\title{
2. Das Verhältnis von Wissen und Können
}

Die Grammatik und die Verwendungsweisen epistemischer Ausdrücke haben sich als sehr facettenreich herausgestellt. Bei ihrer Klassifizierung hat sich gezeigt, dass nicht nur propositionales Wissen allein für die Erkenntnistheorie relevant ist. Auch Können hat kategorialen Status. Im Folgenden wird es darum gehen auszuloten, welche Verhältnismöglichkeiten es für Wissen und Können grundsätzlich geben kann. Ich werde einige wichtige Vorschläge durchgehen und dabei die zunehmende Plausibilität der jeweiligen Verhältnismöglichkeit herausarbeiten. Zunächst werde ich rein formal untersuchen, ob sich eine vollkommene Kongruenz der beiden Kategorien annehmen lässt. Dem schließen sich Überlegungen zum genauen Gegenteil einer vollkommenen Exklusion an. Daraufhin werde ich zu einer hierarchisierenden Betrachtungsweise übergehen und erörtern, ob Können immer Wissen ist. Am Ende jedoch wird die Position, wonach im Gegenteil Wissen immer Können ist, als die überzeugendste übrig bleiben. Damit ist zugleich das Thema des verbleibenden Teils dieser Arbeit abschließend eingeführt. Ich werde die Auffassung vertreten, dass es sich bei dem Verhältnis von Wissen und Können um ein anti-intellektualistisches handelt. Dabei schließe ich nicht aus, dass die Begriffe Kongruenzen aufweisen. Ich strebe allerdings keine begriffliche Reduktion von Wissen auf Können an. Vielmehr geht es mir um den Nachweis eines Fundierungsverhältnisses.

\subsection{Kongruenz und Exklusion}

Gemäß einer eher formalen Überlegung gibt es die Möglichkeit, das Verhältnis von Wissen und Können entweder als eines der vollkommen Kongruenz oder aber der vollkommen Exklusion zu betrachten. Im ersten Fall geht man davon aus, dass es sich um zwei koextensionale Ausdrücke handelt, bei denen es allenfalls Unterschiede idiomatischer oder kontextueller Natur gibt. Im anderen Fall betrachtet man die Ausdrücke „Wissen“ und „Können“ als Bezeichnungen für zwei distinkte und irreduzible epistemische Kategorien. Demnach gibt es keinerlei Übereinstimmung im jeweiligen Begriffsbild. Neben dieser Form der 
Kombinatorik besteht zudem die Möglichkeit, Wissen und Können in ein hierarchisches Verhältnis zu setzen. Berücksichtigt man stärker den begrifflichen Gehalt, so gibt es einerseits die Möglichkeit, Können in Abhängigkeit von Wissen zu sehen. Der Begriff des Könnens wird dann logisch an den des Wissens geknüpft. Etwas zu können, wird auf ein entsprechendes Tatsachenwissen zurückgeführt. Dies ist die Sichtweise des epistemologischen Intellektualismus. Andererseits ist es aber ebenso gut möglich, den Begriff des Wissens logisch an den des Könnens zu knüpfen. Dann behauptet man, dass etwas zu wissen letztlich immer heißt, etwas zu können. Jeder Form von propositionalem Wissen liegt demnach ein Können zugrunde, sei es als eine sensomotorische, eine kognitive oder eine intellektuelle Fähigkeit. Dies ist die Sichtweise des epistemologischen Anti-Intellektualismus.

Die formale lässt sich mit der hierarchischen Kombinatorik erneut in Beziehung setzen. Ein kongruentes Verhältnis von Wissen und Können schließt naturgemäß ein exklusives ebenso aus, wie der Intellektualismus den Anti-Intellektualismus. Grundsätzlich ausschließen lässt sich dagegen nicht, dass eine der ersten beiden Möglichkeiten mit einer der letzten beiden verknüpft und gemeinsam vertreten wird. Tatsächlich geht der Intellektualismus oft mit der Vorstellung Hand in Hand, Wissen und Können seien kongruent. Dagegen tendieren anti-intellektualistische Positionen eher dazu, Wissen und Können als exklusiv zu betrachten. Doch schauen wir uns die einzelnen Verhältnisweisen der Reihe nach an.

Ein Kongruenzverhältnis bei Wissen und Können lässt sich auf semantischem Wege verständlich machen. Man geht davon aus, dass die beiden Ausdrücke sich zwar in der Bedeutung unterscheiden, letztlich aber dieselbe Referenz haben. ${ }^{10}$ Demnach sind die Wörter „Wissen“ und „Können“ lediglich zwei unterschiedliche Art und Weisen, ein und dasselbe zu sagen. Die Bedeutung der beiden Ausdrücke ist verschieden, insofern damit jeweils etwas Spezifische gemeint ist. Das, worauf sie sich letztlich beziehen, ist dabei aber genau das Gleiche. Anders gesagt heben

${ }^{10}$ Die Unterscheidung von Bedeutung und Referenz geht ursprünglich zurück auf G. Freges Überlegungen zu „Sinn“ und „Bedeutung“. Vgl. Frege (1994), 40 ff. Sie entspricht der auf Carnap zurückgehenden Unterscheidung von „Intension“ und „Extension“. Vgl. Carnap (1947). Die Unterscheidung von „Bedeutung“ und „Referenz“ hat W.V.O. Quine etabliert. Vgl. Quine (1960). 
die Ausdrücke lediglich unterschiedliche Aspekte ein und derselben Sache hervor. So gesehen können wir Wissen und Können als kongruent betrachten. Die Frage ist allerdings, was als gemeinsames Referenzobjekt von „Wissen“ und „Können“ gelten soll. Auf Anhieb ist nicht zu sehen, was als ein solcher zusätzlicher Gegenstand der gemeinsamen Referenz in Frage kommt. Ein Kandidat dafür müsste erst noch gefunden oder festgelegt werden.

Etwas anders sieht es aus, wenn gesagt wird, dass der eine der beiden Ausdrücke durch den anderen lediglich erklärt oder gedeutet wird. Wissen beispielsweise gilt dann als dasjenige, was ebenso gut durch das Wort „Können“ zum Ausdruck gebracht wird. Anders herum bezieht sich „Wissen“ gleichbedeutend auf Wissen und Können. Tatsächlich gibt es eine Version des Intellektualismus, die etwas in dieser Art behauptet. Jason Stanley und Timothy Williamson bezweifeln in ihrem Aufsatz „Knowing how “11 , dass es einen bedeutsamen Unterschied zwischen propositionalem Wissen und Können gibt. Sie vertreten die Auffassung, dass sich Können letzten Endes auf Wissen reduziert lässt. Denn bei Können handle es sich genau genommen nur um eine bestimmte „Art des Gegebenseins“ von Wissen. ${ }^{12}$ Dabei gehen sie aber nicht so weit zu behaupten, Können sei komplett mit propositionalem Wissen gleichzusetzen. Insofern ist ihr Ansatz auch weniger ein Beispiel für ein kongruentes Verständnis als für den epistemologischen Intellektualismus. Dazu mehr im zweiten Teil dieses Kapitels.

Eine vollkommene Kongruenz von Wissen und Können halte ich für abwegig. Sowohl Wissen als auch Können findet in unserem Sprachgebrauch eine sehr eigenständige Verwendung, was auf eine zumindest ansatzweise eigenständige kognitive Kompetenz schließen lässt. Ich sehe auch keinen methodischen Vorteil darin, Wissen und Können letztlich als das Gleiche zu betrachten, anstatt tatsächlich von einem Unterschied auszugehen. Die Hypothese der Kongruenz mag prinzipiell vorstellbar sein. Wirklich ernst zu nehmen ist sie aber nicht.

\footnotetext{
${ }^{11} \mathrm{Vgl}$. Stanley/Williamson (2001), 411.

${ }^{12}$ Der Ausdruck „Art des Gegebenseins“ geht ebenfalls zurück auf Frege. Vgl. erneut Frege (1994), $40 \mathrm{ff}$.
} 
Ganz ähnlich verhält es sich, wenn man vom genauen Gegenteil ausgeht und die Ausdrücke „Wissen“ und „Können“ als wechselseitig exklusiv ansieht. Nach dieser Auffassung hat jeder der beiden nicht nur seine ihm eigene Bedeutung, sondern auch seine ganz eigene Referenz. Diese lässt sich jeweils ähnlich scharf abgrenzen, wie es beispielsweise bei den Ausdrücken „Tag“ und „Nacht“" oder „,schwarz“ und „weiß“ möglich ist. Wer nach diesem Verständnis „Wissen“ sagt, kann keinesfalls „Können“ meinen. Auch gegen diese Sichtweise spricht die Art, wie wir diese Ausdrücke üblicherweise verwenden. Eine derart strenge Trennung entspricht nicht der sprachlichen Realität. Schließlich ist es in einigen Kontexten durchaus möglich $\mathrm{zu}$ sagen, dass „etwas $\mathrm{zu}$ können“ gleichbedeutend ist mit ,wissen, wie man es macht“. An der sprachlichen Oberfläche lässt jedenfalls nichts darauf schließen, dass „Wissen“ und „Können“ sich auf zwei vollkommen diskrete Gegenstandsbereiche beziehen.

Ansätze eines solchen, exklusiven Verständnisses finden sich folglich äußerst selten. Gemäß einer ,starken“ Lesart lässt sich Martin Heideggers Unterscheidung von „Zuhandenheit“ und „Vorhandenheit“ als eine solche kategorische Trennung von Können und Wissen interpretieren. ${ }^{13}$ Unter „Zuhandenheit" versteht dieser die Art und Weise, wie Menschen durch den gekonnten, praktischen Umgang mit Gegenständen in die Alltagswelt involviert sind. Dem gegenüber steht die „Vorhandenheit" als eine rein theoretische Auseinandersetzung mit und ein Wissen von den Dingen. Den Übergang von „Zuhandenem“ zu „Vorhandenem“ beschreibt Heidegger als einen diskreten Wechsel von einem Modus in den anderen. ${ }^{14}$ Demnach muss man sich den Menschen als ein Wesen vorstellen, das sich im Alltag durch Können bewährt. In Momenten jedoch, in denen er auf Wissen

${ }^{13}$ Vgl. Heidegger (1993), 69 ff. Zur Unterscheidung einer ,starken“ und „schwachen Lesart" dieser Termini vgl. Brandom (1983b), $405 \mathrm{f}$.

14 Vgl. Heidegger (1993), 61, 69 u. 357 f. Für eine kritische Darstellung des Verhältnisses von „Zuhandenheit“ und „Vorhandenheit“" vgl. auch Prauss (1996). G. Prauss zeigt, dass Heideggers Konzeption des Verhältnisses von Erkennen und Handeln und insbesondere der Übergang vom Handeln zum Erkennen inkonsistent ist. Für die Einschätzung, dass „Zuhandenheit“ ohne Sprache und damit ohne thematisierendes Behandeln von Dingen als „,vorhanden“ nicht denkbar ist vgl. auch Brandom (2002), 45. 
zurückgreift, tritt dieses Können vollständig in den Hintergrund. Wissen und Können sind somit Zustände, in denen sich eine Person nie gleichzeitig befinden kann. Dass diese Sichtweise nicht ohne Probleme ist, sieht Heidegger allerdings selbst. ${ }^{15}$ Er bemerkt die offensichtliche Schwierigkeit, die scharfe Trennlinie zwischen Wissen und Können festzulegen und näher zu bestimmen. Diese Problematik bleibt aber unerörtert.

Andere Konzeptionen, die Wissen und Können scharf voneinander trennen, vermögen ebenso wenig $\mathrm{zu}$ überzeugen. David Carr beispielsweise behauptet, Können lasse sich anhand von drei Bedingungen definieren, welche sich klar von denen unterscheiden, die für propositionales Wissen gelten. ${ }^{16}$ Für ihn ist Können ausschließlich auf praktische Fähigkeiten bezogen und somit klar von Wissen abgegrenzt. Damit entfällt in seinem Modell allerdings die Möglichkeit einer theoretischen Unterweisung in praktische Fähigkeiten vollkommen. Eine Übersetzung von Können in Wissen ist prinzipiell ausgeschlossen. Viele unserer Lernprozesse sind aber gerade darauf angewiesen. Eine strikte Trennung ist daher wenig plausibel.

Wissen und Können sind also zwei epistemische Kategorien, die weder als kongruent noch als exklusiv gelten können. Es liegt daher nahe, ihr Verhältnis zueinander als ein hierarchisches zu begreifen. Wenn zwischen Wissen und Können weder vollständige Übereinstimmung noch strikte Unvereinbarkeit herrscht, dann ist davon auszugehen, dass eine der beiden Kategorien grundlegender ist als die andere. Der Intellektualist behauptet, dass Wissen grundlegender ist als Können. Für ihn steht Können in Abhängigkeit von Wissen. Diese Position werde ich in den folgenden Abschnitten vorstellen und kritisch hinterfragen. Wenn sie sich als unhaltbar herausstellt, wird der Weg frei für die gegenteilige Position, die stattdessen von einem Primat des Könnens ausgeht.

\footnotetext{
${ }^{15}$ Vgl. Heidegger (1993), $357 \mathrm{f}$.

${ }^{16}$ Vgl. Carr (1981).
} 


\subsection{Intellektualismus}

Nehmen wir an, jemand behauptet von sich, er könne Fahrrad fahren. Er bietet an, sein Können unter Beweis zustellen. Wir stellen ihm ein Fahrrad zur Verfügung und fordern ihn auf, damit ein paar Meter zu fahren. Er scheitert allerdings ein ums andere Mal. Später analysieren wir mit ihm sein gescheitertes Bemühen. Wir fragen ihn: „Weißt du denn nicht, dass du den Lenker grade halten und kräftig in die Pedale treten musst, dass du den Blick nach vorne richten und aufrecht sitzen musst?" Indem wir so reagieren, geben wir uns als Vertreter eines epistemologischen Intellektualismus $\mathrm{zu}$ verstehen. Wir sind überzeugt, dass der vermeintliche Fahrradfahrer, will er sein Können realisieren, zuvor eine Liste von Regeln berücksichtigen muss. Wir nehmen an, dass ein bestimmtes Tatsachenwissen die Voraussetzung dafür ist, um über ein entsprechendes Können verfügen zu können.

Philosophen haben den Ausdruck „Intellektualismus“ zur Kennzeichnung der unterschiedlichsten Positionen verwendet. Am wahrscheinlich nachhaltigsten hat den Begriff in seiner epistemologischen Lesart Gilbert Ryle geprägt. ${ }^{17}$ Er versteht darunter eine Reihe von Annahmen, die sich wie folgt zusammenfassen lassen: Es gibt Handlungsweisen, in denen ein Können zum Ausdruck kommt. Diese stellen entweder in sich bereits eine besondere Fähigkeit dar. Hierfür ist das Fahrradfahren ein Beispiel. Oder aber ihre besondere Qualität kommt dadurch zum Ausdruck, dass wir sie als intelligent, überlegt, raffiniert, klug, scharfsinnig, geschickt, logisch etc. bezeichnen. So stellen wir beispielsweise fest, dass jemand scharfsinnig argumentieren oder raffiniert Schach spielen kann. Demnach ist Können also ebenso Ausdruck von Intelligenz wie Tatsachenwissen. Der Intellektualist behauptet nun, dass diese gekonnten Handlungsweisen ihren Anspruch auf Intelligenz dadurch erwerben, dass ihnen eine innere, intellektuelle Planung vorausgeht. Wer intelligent oder gekonnt vorgeht, handelt überlegt und reflektiert. Er denkt, bevor er handelt, insofern er auf bestimmte Wahrheiten zurückgreift, die angeben, wie er sich zu verhalten hat. Intelligent oder gekonnt zu handeln, heißt nach Auffassung des Intellektualisten, zuvor intellektuell tätig

${ }^{17}$ Vgl. Ryle (1945/46) u. Ryle (1949), Kap. 2. Zu einem ähnlichen Verständnis des Begriffs vgl. auch Dewey (1958), $21 \mathrm{ff}$. 
gewesen zu sein. Jedes Stück Können wird durch ein Stück Wissen gestützt. Wer etwas kann, weiß etwas. Und er kann es genau deshalb, weil er etwas weiß. Können oder intelligentes Handeln ist demnach durch Wissen definiert. Die Theorie beziehungsweise das Theoretisieren geht der intelligenten Praxis voraus. Somit lässt sich der Intellektualismus auf den Grundgedanken reduzieren, dass Können eine Art des Wissens ist.

Ryle hält den Intellektualismus für eine unhaltbare „Legende“. Dass Können eine Art des Wissens sein soll, hält er für unlogisch. Vielmehr begreift er es als eine eigenständige epistemische Kategorie und dies zunächst einmal aufgrund der folgenden drei Überlegungen. Erstens sieht er die Eigenständigkeit von Können dadurch gewährleistet, dass wir zwar nach den Gründen für Wissen fragen können, nicht aber nach denen für Können. ${ }^{18}$ Er denkt dabei an Fälle, in denen jemanden beispielsweise zu wissen behauptet, dass es an der Tür geklingelt hat. Ich kann ihn fragen, was seine Gründe für diese Überzeugung sind. Dagegen wäre es unangemessen, nach Gründen für mein Können beispielsweise beim Schach oder bei Finanzinvestitionen zu fragen. Zweitens hält Ryle Wissen und Können deshalb für grundlegend verschieden, weil letzteres partiell sein kann, ersteres dagegen nicht. ${ }^{19}$ Damit ist zum Beispiel gemeint, dass ein gewöhnlicher Schachspieler möglicherweise der Taktik und Strategie eines Weltmeisters folgen kann, während sein Können dabei aber nur zum Teil an das des Weltmeisters heranreicht. Dagegen kann man allerdings nicht behaupten, jemand wisse nur teilweise, dass es an der Tür geklingelt hat. Entweder er weiß dies oder nicht. Und drittens schließlich sieht Ryle einen wichtigen Unterschied darin, dass das Lernen oder Aneignen von Können graduell verläuft, während es bei Wissen „relativ plötzlich“ stattfindet. ${ }^{20}$ Gemeint ist damit, dass es eine gewisse Zeit dauert, bis jemand beispielsweise raffiniert Schach spielen oder kluge Finanzinvestitionen tätigen kann. Verglichen damit vergeht aber nur ein kurzer Augenblick, bis jemand weiß, dass es an der Tür geklingelt hat.

\footnotetext{
${ }^{18}$ Vgl. Ryle (1949), 29. P. Snowdon behauptet, wir können nicht fragen: „Was ist der Grund dafür, dass du weißt, dass $p$ ?““. Vgl. Snowdon (2004), 20. Wir können aber sehr wohl fragen: „Was ist der Grund dafür, dass du glaubst, dass $p$ wahr ist?“ oder „Was macht deine Überzeugung, dass $p$, zu Wissen?“'Ich verstehe Ryle auf diese Weise.

${ }^{19} \mathrm{Vgl}$. Ryle (1949), $57 \mathrm{f}$.

${ }^{20} \mathrm{Vgl}$. ebd., 58.
} 
Diese Überlegungen weisen auf wichtige Indizien für die Eigenständigkeit von Können hin. Allerdings sind hier auch Einwände denkbar. Nehmen wir zunächst das letzte Merkmal, die graduelle Aneignung von Können. Hier lässt sich einerseits anmerken, dass auch die Aneignung von Können gelegentlich ausgesprochen plötzlich vor sich geht. Denken wir etwa an Fälle außergewöhnlicher Begabung. So ist nicht ausgeschlossen, dass jemand beispielsweise auf Anhieb Walzer tanzen oder beim Saxophon spielen in das Zwerchfell atmen kann. Er wird sein Können natürlich wiederholte Male unter Beweis stellen müssen. Dann aber werden wir zugestehen, dass er vom ersten Moment an, ohne einen langen Lernprozess Walzer tanzen oder in das Zwerchfell atmen konnte. Andererseits kann sich der Erwerb von Wissen in manchen Fällen alles andere als plötzlich vollziehen. Acht Jahre vergingen beispielsweise, bis Andrew Wiles wusste, dass Pierre de Fermats Vermutung zu höheren Abwandlungen des Satzes von Pythagoras stimmt. ${ }^{21}$

Auch die Behauptung, dass Können im Unterschied zu Wissen partiell sein kann, ist nicht ohne Einschränkung gültig. Es ist sicher richtig, dass jemand beispielsweise nur zum Teil scharfsinnig argumentieren oder elektrische Geräte reparieren kann, etwa weil er gelegentlich nicht weiter weiß oder einen Fehler macht. Das entsprechende Können ist partiell, geht aber nicht verloren. Man kann sagen, dass es weniger vollständig oder stabil ist, als es sein könnte. ${ }^{22}$ Eine ganz ähnliche Form der Unvollständigkeit ist bei Wissen aber ebenfalls denkbar, etwa wenn eine vermeintliche Tatsache weniger gut oder überzeugend gerechtfertigt ist als andere. Frage ich beispielsweise einen Touristen auf der Straße nach dem schnellsten Weg zum Bahnhof, so werde ich vermutlich mit weniger „vollständigem“ Wissen konfrontiert, als wenn ich einen Taxifahrer oder einen Stadtführer frage.

Daraus lässt sich auch ein Einwand gegen die Annahme ableiten, dass nur bei Wissen nach Gründen gefragt werden kann. Zwar können wir tatsächlich bei Können nicht nach einzelnen, theoretischen Evidenzen oder Beweisen fragen. Da es aber durchaus in vergleichbarer Weise an

${ }^{21}$ Gemeint ist der Beweis der Vermutung Fermats, wonach die Gleichung $a^{n}+b^{n}=c^{n}$ für ganzzahlige $\mathrm{a}, \mathrm{b}, \mathrm{c} \neq 0$ und natürliche Zahlen $\mathrm{n}>2$ keine Lösung besitzt. Vgl. Wiles (1995).

${ }^{22} \mathrm{Vgl}$. Hetherington (2006), 92. 
Bedingungen geknüpft ist, können wir für Können etwas in der Art fragen wie: „Was ist der Beleg dafür, dass $S q$ kann?“ So wie man für Wissen zur Bedingung machen kann, dass es eine Begründung gibt, so kann man für Können beispielsweise zur Bedingung machen, dass das Gekonnte erfolgreich wiederholt wird. ${ }^{23}$ Dem Kriterium der Begründung bei Wissen lässt sich also das Kriterium des Erfolgs bei Können gegenüberstellen. Daraus ergibt sich dann auch eine weitere Erklärung dafür, warum jemand etwas sowohl partiell können als auch wissen kann. Jemand kann beispielsweise nur zum Teil Ski fahren können, weil er dabei nicht vollständig erfolgreich ist. Und jemand kann nur zum Teil wissen, dass beispielsweise der schnellste Weg zum Bahnhof über die Torstraße führt, weil er dies nicht vollständig begründen kann.

Die Überlegungen, die den kategorialen Unterschied von Wissen und Können belegen sollen, gehören zum weniger überzeugenden Teil in Ryles Kritik des Intellektualismus. ${ }^{24}$ Damit allein lässt sich die Behauptung, Können sei ein Art des Wissens, nicht unstrittig widerlegen. Ryles stärkster und auch bekanntester Einwand gegen den Intellektualismus ist allerdings auch ein anderer. In einem Reductio-Argument macht er deutlich, dass dieser letztlich in einen unendlichen Regress führt. Er schreibt:

According to the [intellectualist] legend, whenever an agent does anything intelligently, his act is preceded and steered by another internal act of considering a regulative proposition appropriate to his practical problem. (...) Next, supposing still that to act reasonably I must first perpend the reason for so acting, how am I led to make a suitable application of the reason to the particular situation which my action is to meet? (...) By the original argument, therefore, our intellectual planning process must inherit its title to [intelligence or] shrewdness from yet another interior process of planning to plan, and this process could in its turn be [intelligent] or shrewd. The regress is infinite, and this reduces to absurdity the theory that for an operation to be intelligent it must be steered by a prior intellectual operation..$^{25}$

${ }^{23}$ Vgl. hierzu auch Carr (1981), 58. Für D. Carr ist eine der drei Bedingungen für Können die des ,erkennbaren Erfolgs“.

${ }^{24}$ Ich verzichte auf die Diskussion weiterer, ebenso wenig überzeugender Einwände. Vgl. z. B. Ryle (1945/46), 5 u. 16 u. Ryle (1949), 30 f. Vgl. auch White (1982), 26.

${ }^{25}$ Ryle (1949), 31 f. (Meine Ergänzung und Änderung, A. S.). Vgl. parallel dazu auch Ryle (1945/46), 2 f. Ryles Regressargument hat große Ähnlichkeit mit L. 
Was der Intellektualist behauptet, ist absurd, weil er einen Prozess voraussetzt, der genau genommen nie an ein Ende gelangt. Denn derjenige, der gekonnt oder intelligent handelt, müsste zuvor stets die entsprechenden „regulativen Propositionen“26 berücksichtigen. Er müsste entscheiden, genau welches Wissen für sein Können das richtige ist. Dieser evaluative Denkvorgang wäre aber ebenfalls etwas, das intelligent oder dumm ausgeübt werden kann. Für die Wahl der richtigen Propositionen wäre also ein weiterer evaluativer Denkvorgang nötig, bei dem erneut Propositionen berücksichtigt werden müssten und so immer weiter. Eine intelligente oder gekonnte Handlung würde unter dieser Voraussetzung nie zur Ausführung kommen, weil ihr eine unendliche Reihe evaluativer Denkvorgänge vorgeschaltet wäre.

Viele Autoren halten den Intellektualismus durch Ryles Regressargument für erfolgreich widerlegt. ${ }^{27}$ Es gibt dagegen allerdings auch Einwände. Zuletzt hat Jason Stanleys und Timothy Williamsons Aufsatz „Knowing How" großes Aufsehen erweckt und eine rege Diskussion ausgelöst. ${ }^{28}$ Daher werde ich darauf im Folgenden näher eingehen. Die beiden Autoren versuchen im ersten Teil ihres Aufsatzes, Ryles Regressargument zu widerlegen. Der zweite Teil dient einer Rehabilitierung des Intellektualismus. Dabei beziehen sie sich explizit auf moderne Intellektualisten wie David Brown, Jaakko Hintikka oder Carl Ginet. ${ }^{29}$ Somit liegt mit diesem Aufsatz eine sehr aktuelle Version dieser Sichtweise vor, die zugleich die eigene Tradition aufgreift. Wenn es gelingt, diesen Rehabilitierungsversuch als inkohärent auszuweisen, dann ist der Intellektualismus insgesamt als plausibles Erklärungsmodell für das Verhältnis von Wissen und Können zurückgewiesen. Als beste Erklärung

Wittgensteins Regelregressargument sowie mit L. Carroll Regress des Konditionals. Ryle bezieht sich explizit auf letzteres. Vgl. auch Kap. 3.5 u. 5.12.

26 Ich übernehme hier die Rede von „Propositionen“, obwohl ich die von „Überzeugungen“ für angemessener halte und später auch zu dieser Redensweise übergehen werde. Vgl. Kap. 4.

${ }^{27}$ Vgl. z. B. Carr (1981), 55, Craig (1990), 154 o. Snowdon (2004), 14 ff.

${ }^{28} \mathrm{Zu}$ den Reaktionen auf diesen Aufsatz vgl. z. B. Schiffer (2002), Koethe (2002), Rumfitt (2003), Rosefeldt (2004), Noë (2005), Hetherington (2006), Gochet/de Rouilhan (2007) o. Jung/Newen (2010).

${ }^{29}$ Vgl. Brown (1970), Hintikka (1972) u. Ginet (1975). 
bleibt dann der Anti-Intellektualismus übrig. Doch zunächst zu den Einwänden gegen Ryles Regressargument.

Stanley und Williamson rekonstruieren Ryles Argument, indem sie es auf zwei allgemeine Prämissen und eine Reductio-Annahme reduzieren. ${ }^{30}$ Die erste Prämisse besagt, dass das Ausführen einer Handlung $F$ darauf verweist, dass $F$ gekonnt wird. ${ }^{31}$ Die zweite Prämisse besagt, dass die Anwendung des Wissens, dass $p$ dem intellektuellen Erwägen der Proposition $p$ gleichkommt. In der Reductio-Annahme (RA) schließlich kommt die Grundüberzeugung des Intellektualismus zum Ausdruck, wonach dem Können von $F$ das Wissen um eine entsprechende Proposition $\phi(F)$ entspricht. Eine solche wäre ein Satz der Form: Die Proposition $p$ ist die Voraussetzung für die Handlung $F$.

Zusammengenommen führen die beiden Prämissen und die ReductioAnnahme $\mathrm{zu}$ einem Widerspruch. Schließlich ist das intellektuelle Erwägen einer Proposition selbst eine Handlung. Die erste Prämisse muss also auch für die in der zweiten Prämisse beschriebene Handlung des intellektuellen Erwägens der Proposition $p$ gelten. Wenn gemäß der intellektualistischen Grundüberzeugung (RA) Können Wissen und gemäß der zweiten Prämisse Wissen das intellektuelle Erwägen einer Proposition ist, so enden wir erneut bei der ersten Prämisse. Es entsteht immer wieder eine weitere Handlung des intellektuellen Erwägens und daraus wiederum eine und so weiter ad infinitum. Wir geraten in einen infiniten Regress intellektueller Erwägungen. Demnach kann eine Handlung $F$ überhaupt nicht ausgeführt werden, da sie eine solche unendliche Anzahl von Erwägungen voraussetzt. Zumindest ist das die Konsequenz, wenn sowohl die beiden Prämissen als auch die Reductio-Annahme wahr sind. Es ist aber zweifelsohne so, dass wir Handlungen ausführen. Mindestens eine der drei Thesen muss daher falsch sein. Für Ryle ist es bekanntlich die intellektualistische Grundüberzeugung (RA), weshalb er dafür plädiert, sie aufzugeben.

Um nun die intellektualistische Überzeugung, dass Können eine Art des Wissens ist, beibehalten zu können, richtet sich Stanleys und

\footnotetext{
${ }^{30} \mathrm{Vgl}$. Stanley/Williamson (2001), $413 \mathrm{f}$.

31 Die Notation „Fen können“ entspricht meiner bisherigen Formalisierung , $q$ können“.
} 
Williamsons Kritik gegen die erste und zweite Prämisse. Ihre Vorgehensweise ist simpel und erfolgt in zwei Schritten. Zunächst stellen sie fest, dass es Handlungen gibt, die kein Können zum Ausdruck bringen. Als Beispiele nennen sie das Verdauen von Nahrung oder das Gewinnen in einer Lotterie. ${ }^{32}$ Beides, so stellen sie fest, kann man nicht können. Soll also die erste Prämisse gültig bleiben, so muss sie auf intentionales Handeln eingeschränkt werden. Auf diese Weise können zweifelhafte Fälle wie diese als Beispiele für $F$ ausgeschlossen werden. Dieses Zugeständnis wirkt sich allerdings vernichtend auf die zweite Prämisse aus. Im zweiten Schritt stellen die beiden Autoren nämlich fest, dass es auch Fälle von Wissen gibt, mit denen kein intentionales Erwägen von Propositionen einhergeht. Sie geben folgendes Beispiel: Jemand bekundet sein Wissen, dass sich die Tür durch Drehen des Türgriffs öffnen lässt, indem er den Türgriff ganz automatisch dreht, ohne dabei irgendeine Proposition in Erwägung zu ziehen. ${ }^{33}$ Um die zweite Prämisse beibehalten zu können, schlagen sie daher vor, das Erwägen von Propositionen als eine Handlung in einem eingeschränkten, nicht-intentionalen Sinn zu verstehen. Das wiederum bedeutet aber, dass diese Art Handlung nicht in die erste Prämisse eingesetzt werden kann. Um deren Plausibilität zu garantieren, wurde sie ja gerade auf intentionales Handeln eingeschränkt. Das Ergebnis dieser Überlegungen soll sein, dass es keine einheitliche Lesart des Arguments gibt. Offensichtlich können nicht beide Prämissen ihre Gültigkeit behalten. Damit glauben Stanley und Williamson gezeigt zu haben, dass Ryles Regressargument nicht haltbar ist.

Ich halte diese Rekonstruktion des Arguments jedoch in wichtigen Punkten für fehlerhaft und ungenau. Das Beispiel vom Verdauen von Nahrung etwa ist vollkommen abwegig. Niemand kann Nahrung verdauen, nicht weil dies kein intentionaler Akt ist, sondern weil es überhaupt keine Handlung darstellt. Verdauen ist ein organischer Vorgang, der in uns vor sich geht. Analog verhält es sich mit dem Gewinnen in einer Lotterie. Auch dies ist keine Form des Handelns, weder im intentionalen noch in einem anders gearteten Sinne. Daher habe ich derartige Vorgänge und Ereignisse auch bewusst aus der Bestimmung von Können

\footnotetext{
${ }^{32} \mathrm{Vgl}$. Stanley/Williamson (2001), $414 \mathrm{f}$.

${ }^{33}$ Vgl. ebd., 415. Stanley und Williamson zitieren dieses Beispiel aus Ginet (1975), 7.
} 
ausgeschlossen. ${ }^{34}$ In einer Lotterie zu gewinnen, ist ein Ereignis, das mir widerfährt. Was ich allerdings beabsichtigen und können kann, ist, an einer Lotterie teilzunehmen. Ich tue dies, indem ich ein Los erwerbe. Nur in dieser trivialen Hinsicht könnte man sagen, dass ich in einer Lotterie gewinnen „kann“. Dabei jedoch handelt es um die Art von Können, die ich als epistemologisch irrelevant ausgeklammert habe. Denn Können bedeutet hier ,in der Lage sein, etwas zu tun“. Was uns interessiert, ist aber vielmehr Können oder Wissen-wie als eine Fähigkeit oder Kompetenz. Die Beispiele stellen sich also als nicht zutreffend heraus und können nicht als gültige Einwände gegen das Regressargument gelten. Die erste Prämisse ist von ihnen nicht betroffen.

Man kann jedoch fragen, ob die Prämisse in dieser Formulierung überhaupt zutreffend ist. Denn genau genommen gibt sie Ryles Gedanken nur verkürzt und ungenau wieder. Schließlich zeigt die zitierte Passage, dass er nicht jede beliebige Handlung als Können bezeichnet. Vielmehr geht es ihm um solche, die wir implizit oder explizit als intelligent charakterisieren. ${ }^{35}$ Folglich muss die erste Prämisse richtigerweise beinhalten, dass das Ausführen einer als intelligent zu bezeichnenden Handlung $F$ darauf verweist, dass jemand $F$ en kann beziehungsweise weiß, wie man $F$ t. Und in dieser Lesart lässt sie sich problemlos mit der zweiten Prämisse verbinden. Denn es ist durchaus gerechtfertigt, das Erwägen von Propositionen als eine Art intelligente Handlung $\mathrm{zu}$ charakterisieren. Wissen, verstanden als das Erwägen von Propositionen, kommt daher als ein Beispiel für $F$ sehr wohl in Frage. Auch dies stützt Ryles Regressargument.

Was aber, wenn propositionales Wissen auch ohne das Erwägen von Propositionen auskommt? Für Stanley und Williamson ist die zweite Prämisse genau aus diesem Grund falsch. Sie behaupten, dass es häufig „Manifestationen von Wissen“ gibt, die nicht mit dem Erwägen von Propositionen einhergehen. ${ }^{36}$ Die Rede von „Manifestationen“ ist hier allerdings irreführend. Es ist sicher richtig, dass sich das Erwägen einer entsprechenden Proposition nicht immer manifestiert, wenn jemand etwas

\footnotetext{
${ }^{34}$ Vgl. Kap. 1.2. Vgl. ebenso Noë (2005), 279 u. Hetherington (2006), 74.

${ }^{35}$ Vgl. erneut Ryle (1945/46), 2 f. u. Ryle (1949), 29 ff.

${ }^{36}$ Vgl. Stanley/Williamson (2001), 415.
} 
bestimmtes weiß. Dass wir diesen Vorgang nicht immer erkennen können, beweist allerdings nicht, dass es ihn tatsächlich auch nicht gibt. Prinzipiell sind die Manifestation und das Vorkommen eines Vorgangs zwei verschiedene Dinge. ${ }^{37}$ Nicht selten gehen kognitive oder mentale Akte unbewusst oder unbeobachtet vor sich, so auch das Erwägen von Propositionen. Es ist daher durchaus möglich, dass jemand sein propositionales Wissen mehr oder weniger automatisch anwendet. Er verfügt dann über Wissen, ohne jedoch eine entsprechende Proposition bewusst in Erwägung zu ziehen. Man kann auch sagen, er habe unbewusst eine entsprechende Überzeugung. Propositionales Wissen ohne jede Proposition lässt sich jedenfalls nicht leicht plausibilisieren. ${ }^{38}$ Dagegen ist die Annahme des unbewussten Erwägens von Propositionen mit Ryles Argumentation absolut konform.

Somit lässt sich feststellen, dass das Regressargument von Stanleys und Williamsons Einwänden nicht in Zweifel gezogen wird. Es ist ein Irrtum anzunehmen, organische Vorgänge oder zufällige Ereignisse seien Handlungen, die man können kann. Ebenso ist es ein Irrtum anzunehmen, mit Können meinten wir jede nur erdenkliche Handlung. Ryle bezieht Können bewusst auf Handeln, das wir als intelligent, überlegt, raffiniert, klug, scharfsinnig, geschickt, logisch etc. bezeichnen. Und es ist ein Irrtum anzunehmen, propositionales Wissen sei ohne das Erwägen von Propositionen möglich. Entweder jemand weiß, dass das Drehen des Türgriffs die Tür öffnet. Dann erwägt er zumindest unbewusst die Proposition, dass dies so ist. Oder wir begreifen das Türöffnen als eine Art automatisierten Vorgang oder Reflex. Dann kann aber auch nicht von propositionalem Wissen die Rede sein. Jedenfalls ist ,automatisches Handeln“ kein Beispiel für die „Manifestation“ von propositionalem Wissen ohne das Erwägen von Propositionen.

\subsection{Versuch einer Rehabilitierung des Intellektualismus}

Im Anschluss an ihre Kritik formulieren Stanley und Williamson eine Version des Intellektualismus, die sie insbesondere durch Erkenntnisse der

\footnotetext{
${ }^{37}$ Vgl. Noë (2005), 281.

${ }^{38} \mathrm{Zu}$ hiervon abweichenden Auffassungen vgl. Anm. 153 u. 156.
} 
modernen Sprachwissenschaft zu belegen versuchen. ${ }^{39}$ Dabei beschäftigen sie sich vorwiegend mit der Grammatik englischer Wissensausdrücke. Die sehr technische Ausrichtung ihres Ansatzes unterscheidet sich stark von Ryles dem Common Sense verpflichteten Überlegungen. Es muss daher davon ausgegangen werden, dass der Regresseinwand nicht greift. Im Anschluss an eine kurze Darstellung werde ich erörtern, ob der Intellektualismus in dieser Form als rehabilitiert gelten kann.

Eine zentrale Behauptung der beiden Autoren ist die, dass alle Sätze, die das englische Verb „to know“ beinhalten und durch einen Nebensatz ergänzt werden, eine Relation zwischen einer Person und einer Proposition zum Ausdruck bringen. ${ }^{40}$ Bei ,know that“ ist dies ganz offensichtlich der Fall. Diese Formulierung wird stets durch eine Proposition ergänzt. Dass dies in vergleichbarer Weise auch für Sätze gilt, die die Formulierung „know how" beinhalten, liegt für Stanley und Williamson in deren allgemeiner Struktur begründet. ${ }^{41}$ Sie sehen in Sätzen dieser Art stets eine indirekte Frage enthalten. Demzufolge beinhaltet der Satz „Hannah knows how to ride a bicycle.“ die Frage „How to ride a bicycle?" oder besser „How should she/one ride a bicycle?“42 Das Wissen, das darin zugeschrieben wird, besteht in einer Antwort auf diese Frage. Der Satz ist also genau dann wahr, wenn es mindestens eine Proposition gibt, mit der Hannah diese Frage beantworten kann. Somit scheint das Wissen, das in derartigen Sätzen vorkommt, ebenso von propositionaler Art zu sein, wie jenes in Sätzen mit Know-that-Formulierungen. Der Unterschied ist lediglich, dass die entsprechenden Propositionen in Sätzen mit Know-howFormulierungen nicht immer genau identifiziert werden können.

Wie lässt sich nun das Spektrum der Antworten, das sich hinter Know-how-Sätzen verbirgt, näher eingrenzen? $\mathrm{Zu}$ diesem Zweck schränken Stanley und Williamson den Bereich der möglichen Propositionen ein. Sie legen fest, dass es in Know-how-Sätzen um die Propositionen geht, die Art und Weisen des Ausführens von Handlungen

39 Stanley und Williamson beziehen sich v. a. auf Karttunen (1977) und Groenendijk/Stokhof (1982).

${ }^{40}$ Vgl. Stanley/Williamson (2001), $430 \mathrm{f}$.

${ }^{41}$ Sätze, die durch ein Nomen oder eine Nominalphrase ergänzt werden wie z. B. „She knows Fred.“, sind von dieser Überlegung ausgeschlossen.

${ }^{42}$ Vgl. Stanley/Williamson (2001), $424 \mathrm{f}$. 
enthalten. ${ }^{43}$ Jedem Ereignis - und damit auch jeder Handlung - kommt eine bestimmte Art und Weise zu, auf die es stattfindet. Und die indirekte Frage eines Know-how-Satzes (How should one $F$ ?) fragt nach eben dieser Art und Weise. Das Subjekt des Satzes weiß darauf eine Antwort. So weiß Hannah im erwähnten Beispielsatz von einer Proposition, die eine Art und Weise des Fahrradfahrens beinhaltet. Etwas ausführlicher bedeutet das, dass es eine Art und Weise $w$ gibt, die für Hannah eine Art und Weise ist, Fahrrad zu fahren, und Hannah weiß, dass $w$ eine Art und Weise für sie ist, Fahrrad zu fahren. Dies ist nach Stanley und Williamson die Auflösung des ursprünglichen Satzes, der die Formulierung ,knows how“ enthält.

Entscheidend für diesen Ansatz ist nun die zusätzliche Annahme, dass von einer Proposition auf unterschiedliche „Arten des Gegebenseins“ (modes of presentation) Gebrauch gemacht wird. ${ }^{44}$ Gottlob Freges Idee der modalen Bestimmtheit von Bezeichnungen und Propositionen veranschaulichen sie durch Sätze, in denen Überzeugungen zum Ausdruck kommen. Betrachten wir die folgenden Beispiele:

(1) John ist der Überzeugung, dass dieser Mann eine brennende Hose hat.

(2) John ist der Überzeugung, dass er selbst eine brennende Hose hat. ${ }^{45}$

Unter der Voraussetzung, dass sich „dieser Mann“ auf John bezieht, bringen die beiden Nebensätze die gleiche Proposition zum Ausdruck. Der Unterschied liegt Stanley und Williamson zufolge lediglich in der jeweiligen Art des Gegebenseins. So wird John die Überzeugung in (1) auf eine „demonstrative Art des Gegebenseins“ (demonstrative mode of presentation) zugeschrieben. In (2) dagegen wird sie ihm auf eine „Erste Person Art des Gegebenseins“ (first-personal mode of presentation) zugeschrieben.

Analog zu diesem Beispiel konstruieren Stanley und Williamson einen Fall, in dem Wissen zugeschrieben wird. Wieder geht es um Hannah. Mit Verweis auf eine Person, die in ihrer Nähe Fahrrad fährt, wird ihr gesagt: „Dies ist für dich eine Art und Weise, Fahrrad zu fahren.“ In diesem

\footnotetext{
${ }^{43}$ Vgl. ebd., 427.

${ }^{44}$ Vgl. ebd. Zur „Art des Gegebenseins“ vgl. auch erneut Frege (1994), 40 ff.

${ }^{45}$ Vgl. Stanley/Williamson (2001), 428. (Meine Übersetzung, A. S.)
} 
Zusammenhang lassen sich, so die beiden Autoren, folgende Sätze formulieren:

(3) Hannah weiß, dass diese Art und Weise für sie eine Art und Weise ist, Fahrrad zu fahren.

(4) Hannah kann Fahrrad fahren beziehungsweise weiß, wie man Fahrrad fährt. ${ }^{46}$

„Diese Art und Weise“ soll sich auf die Art und Weise derjenigen Person beziehen, die in Hannahs Nähe Fahrrad fährt. Es handelt sich dabei tatsächlich um eine Art und Weise für sie, Fahrrad zu fahren. Nach Auffassung Stanleys und Williamsons schreiben nun beide Sätze Hannah dieselbe Proposition zu. Wie im vorherigen Fall unterscheiden sie sich ebenfalls lediglich in der Art des Gegebenseins. Während (3) Hannahs Wissen auf eine demonstrative Art des Gegebenseins zum Ausdruck bringt, drückt es (4) auf eine ,praktische Art des Gegebenseins“ (practical mode of presentation) aus. ${ }^{47}$ Indem wir Hannah zuschreiben, dass sie Fahrrad fahren kann, schreiben wir ihr indirekt ein entsprechendes propositionales Wissen auf eine praktische Art und Weise zu. Dies ist im Wesentlichen Stanleys und Williamsons Versuch, den Intellektualismus zu rehabilitieren. Im verbleibenden Teil dieses Kapitels wird es darum gehen, ihn einer kritischen Überprüfung zu unterziehen.

\subsection{Wissenssätze und indirekte Fragen}

Schauen wir uns zuerst die Überlegungen zu indirekten Fragen in Wissenssätzen an. Hier sind Stanleys und Williamsons sprachwissenschaftliche und grammatische Untersuchungen nur bedingt aussagekräftig. Das zeigt sich, wenn sie auf andere Sprachen als das Englische ausgeweitet werden. ${ }^{48}$ Dann nämlich stellt sich die Frage, ob Satzkonstruktionen analog „know that" und „know how“ tatsächlich immer eine Relation zwischen einer Person und einer Proposition zum Ausdruck bringen. Das deutsche Verb „können“ etwa gibt weder einen Hinweis auf eine indirekte Frage noch auf eine Proposition. Sage ich beispielsweise, dass jemand

\footnotetext{
${ }^{46}$ Vgl. ebd. (Meine Übersetzung und geringfügige Vereinfachung, A. S.)

${ }^{47}$ Vgl. ebd., 429.

${ }^{48} \mathrm{Zu}$ den folgenden Überlegungen vgl. auch Rumfitt (2003).
} 
scharfsinnig argumentieren oder raffiniert Schach spielen kann, so drücke ich eine Relation zwischen einer Person und der Handlung des Argumentierens oder Schachspielens aus. Die Tatsache, dass es im Deutschen neben der Formulierung „wissen, wie“ die Formulierung „können“ gibt, verbietet somit die generelle Annahme einer PersonProposition-Relation in Wissens- beziehungsweise Könnenssätzen.

Das Französische unterstützt diesen Einwand. Hier gibt es zum einen die Formulierung ,savoir comment“, die dem englischen „know how“ und dem deutschen „wissen, wie“ entspricht. Damit lässt sich beispielsweise sagen: „Elle sait comment déstabiliser son adversaire.“ (Sie weiß, wie man seinen Gegner verunsichert.) Daneben wird „savoir" aber auch ohne das Interrogativadverb „,comment“ verwendet. Diese Verwendungsweise taucht in Sätzen auf wie „Elle sait nager.“ (Sie kann schwimmen.) Sie entspricht dem deutschen „können“, während sie im Englischen ebenfalls mit „know how“ übersetzt wird. Wie „können“ auch lässt diese Verwendungsweise von „savoir“ keinerlei Rückschlüsse auf Propositionen zu, die als Antworten auf indirekt gestellte Fragen dienen könnten. Diese Eigenart der französischen Sprache stellt Stanleys und Williamsons Behauptung also ebenfalls in Frage.

Man könnte nun annehmen, dass es sich bei einem Satz wie „Elle sait nager." um eine unvollständige Satzform handelt, die der Formulierung „Elle sait comment nager.“ entspricht. Die beiden Sätze können aber nicht ohne weiteres gleichgesetzt werden. Genau genommen ist letzterer nämlich kein wohlgeformter Satz des Französischen. ${ }^{49}$ Wenn den Formulierungen etwas gemein ist, dann bestenfalls der Hinweis auf dasselbe Können. Generell drücken französische Wissenssätze ohne Interrogativadverb allgemeine, nicht weiter spezifizierte sensomotorische oder intellektuelle Fähigkeiten aus. Das entspricht einem allgemeinen, nicht weiter spezifizierten Können, so wie ich es im letzten Kapitel eingeführt habe. In Sätzen mit Interrogativadverb geht es dagegen um ganz bestimmte Probleme oder um die Art und Weise, wie etwas gemacht wird. Hier ist das Können möglicherweise weiter spezifiziert. Sätze wie „Elle sait comment nager.“ erhalten daher zumeist eine Ergänzung der Art: „Elle sait comment nager vite dans des compétitions." (Sie weiß, wie man in Wettkämpfen

${ }^{49}$ Vgl. ebd., 161 f. u. Gochet/de Rouilhan (2007), 30. 
schnell schwimmt.) In Fällen wie diesen könnte man eine indirekte Fragekonstruktion („Comment nager vite dans des compétitions?“) entdecken und einen Verweis auf propositionales Wissen vermuten. Entscheidend ist jedoch, dass dies für das entsprechende Können vollkommen irrelevant ist. Ob jemand schwimmen kann, hängt nicht davon ab, ob er in Wettkämpfen schnell schwimmen kann.

Doch nicht nur im Deutschen und Französischen wird auf ein Interrogativadverb verzichtet, wenn von Können die Rede ist. Auch im Griechischen und Lateinischen gibt es Formulierungen, in denen sensomotorische oder intellektuelle Fähigkeiten ausgedrückt werden, ohne dass darin eine indirekte Frage vorkommt. ${ }^{50}$ Und im Russischen gibt es für Fälle von „know how“ überhaupt keine entsprechende Formulierung. Dem Können und Wissen-wie entspricht hier jeweils ein eigenes Verb (уметь). Allerdings wird dies weder durch einen propositionalen Nebensatz noch durch eine indirekte Frage, sondern ausschließlich durch einen Infinitiv ergänzt.

Offensichtlich lässt sich also anhand von Sätzen, in denen Wissen zugeschrieben wird, nicht zeigen, dass es darin generell um die Relation einer Person zu einer Proposition geht. Das Englische ist hier keineswegs paradigmatisch. Was dort die Formulierung „know how“ ausdrückt, wird in anderen Sprachen wie etwa dem Deutschen oder Französischen durch Satzstrukturen ohne Fragepronomen übersetzt. Folglich ist in den entsprechenden Satzkonstruktionen auch keine indirekte Frage enthalten. Ebenso wenig lässt sich feststellen, dass indirekte Fragen in Wissenssätzen grundsätzlich auf eine propositionale Tiefenstruktur hinweisen. Hierbei handelt es sich um nicht mehr als eine Möglichkeit, die aber auch eine Besonderheit der englischen Sprache ist.

\subsection{Können als Wissen von Art und Weisen des Ausführens von Hand- lungen}

Der nächste Schritt in Stanleys und Williamsons intellektualistischem Ansatz besteht in der Behauptung, Können sei propositionales Wissen von Art und Weisen des Ausführens von Handlungen. Jemand, der etwas kann,

\footnotetext{
${ }^{50} \mathrm{Vgl}$. auch für das folgende Beispiel Rumfitt (2003), $163 \mathrm{f}$.
} 
weiß demnach kurz gesagt von einer bestimmten Art und Weise zu handeln. Dieser Punkt ist weitestgehend unabhängig von den Überlegungen $\mathrm{zu}$ indirekten Fragen und nicht auf deren Gültigkeit angewiesen. Dass sie nicht haltbar sind, hat keine weiteren Konsequenzen. Ich werde die aufgezeigten Probleme der letzten Abschnitte daher nicht weiter berücksichtigen und diesen nächsten argumentativen Schritt unabhängig davon betrachten.

Die Rede von Art und Weisen zu handeln im Zusammenhang mit Können ist ein beliebtes Element des Intellektualismus. ${ }^{51}$ Das Problem ist jedoch, dass sich ein solcher Zusammenhang gar nicht so leicht identifizieren lässt. Denn eine Rückführung von Können auf Wissen in diesem Sinne ist entweder zu allgemein oder zu speziell. Entweder es wird lediglich die Existenz irgendeiner Art und Weise zu handeln proklamiert, ohne dass klar ist, ob und wie die handelnde Person dazu in Relation steht. Oder aber es wird auf eine ganz bestimmte, kontextuell gegebene Art und Weise Bezug genommen. Hier fehlt dann die allgemeine Gültigkeit, die der Spezifizierung von etwas als Können eignet. Es ist daher fraglich, ob das gemeinte Können überhaupt auch in anderen Situationen als der spezifizierten möglich ist.

Zunächst zu dem Fall, in dem diese Bestimmung zu allgemein ist. Ich will unter Bezug auf Art und Weisen zum Ausdruck bringen, dass Hannah Fahrrad fahren kann. Ich sage daher, sie wisse eine Art und Weise, Fahrrad $\mathrm{zu}$ fahren. (Aus didaktischen und dialektischen Gründen verwende ich „wissen“ hier auf diese etwas eigenwillige Weise.) Dadurch stelle ich nichts weiter fest, als dass es irgendeine Art und Weise gibt, Fahrrad zu fahren, von der Hannah weiß. Das ist insofern legitim, als es natürlich prinzipiell sein kann, dass Hannah auch nur auf eine einzige Art und Weise Fahrrad fahren kann. Es ist aber überhaupt nicht gesagt, dass Hannahs Können tatsächlich dem Wissen um diese eine Art und Weise entspricht. Was garantiert denn, dass es gerade dies ist, was sie zum Fahrradfahren befähigt? Es ist doch gut möglich, dass sie von einer Art und Weise, Fahrrad zu fahren, weiß und trotzdem nicht Fahrrad fahren kann. Man kann sich dies so vorstellen, dass Hannah auf die Frage „Kannst Du Fahrrad fahren?“ mit dem Satz antwortet: „Ja, ich weiß eine Art und

${ }^{51}$ Vgl. z. B. Hintikka (1972), 114 f., White (1982), 11 u. 22 o. Snowdon (2004), 26 ff. 
Weise, Fahrrad zu fahren.“ Diese Antwort geht offensichtlich an der Frage vorbei.

Wichtiger noch ist aber die Frage, ob ich Hannah auf diese Weise überhaupt propositionales Wissen zuschreibe? Dies ist nämlich keineswegs der Fall. Vielmehr handelt es sich bei dem Resultat meiner Reformulierung ihres Könnens um eine Form von Kenntnis. Hannah kennt eine Art und Weise, Fahrrad zu fahren. Sie ist mit dieser Art und Weise vertraut und hat sie verinnerlicht. Ob sie aber über ein entsprechendes Wissen verfügt, ist damit nicht gesagt. Es ist nicht einmal gesagt, dass sie die Art und Weise, Fahrrad zu fahren, die sie kennt, genau identifizieren und sprachlich fixieren kann. Um sinnvoll von propositionalem Wissen $\mathrm{zu}$ sprechen, müsste sie dies aber. Es ist sicher richtig, dass sowohl Wissen als auch Kenntnis Handlungsweisen zum Gegenstand haben kann. Dies lässt sich aber nicht ohne weiteres vergleichen. Schließlich bezieht sich Wissen immer auf Propositionen. Kenntnis dagegen richtet sich auf Gegenstandsbeschreibungen. Folglich kann das, was jemand weiß, wahr oder falsch sein. Von dem, was jemand kennt, würden wir dies jedoch nicht sagen. Wenn sich also Wissen und Kenntnis auf Art und Weisen zu handeln beziehen, dann auf sehr unterschiedliche Weise.

Die Bestimmung von Können als Wissen von Art und Weisen zu handeln läuft also Gefahr, zu allgemein zu bleiben. Wie sich schnell zeigt, ist die einzige Alternative dazu allerdings eine viel $\mathrm{zu}$ spezielle Eingrenzung. Soll gezeigt werden, dass Können propositionales Wissen ist, so muss das Identifizieren bestimmter, angemessener Art und Weisen möglich sein. Die meisten Autoren führen daher eine Art der Bezugnahme in ihr intellektualistisches Modell ein. Alan White beispielsweise behauptet, Können entspreche Wissen derart, dass „das und das“ eine „,unerwähnte“ Art und Weise ist, entsprechend zu handeln. ${ }^{52}$ Paul Snowdon vertritt die Auffassung, Können bedeute zu wissen, dass eine „bestimmte“ Art und Weise diejenige ist, auf die das Gekonnte getan wird. ${ }^{53}$ Allerdings ist auch hier alles andere als klar, wie man sich diese Bezugnahmen konkret vorzustellen hat.

\footnotetext{
${ }^{52} \mathrm{Vgl}$. White (1982), 11 u. 23.

${ }^{53}$ Vgl. Snowdon (2004), 26.
} 
Stanley und Williamson versuchen, der Vagheit solcher Ausdrücke auszuweichen, indem sie ein komplexes und vermeintlich vollständiges Szenario entwerfen. Dabei gehen sie davon aus, dass die geeignete Art und Weise zu handeln letztlich durch ein Demonstrativpronomen identifiziert werden kann. Dazu ist es einerseits notwendig, dass sie im Kontext der Person, die etwas kann, präsent ist. Andererseits muss sie sich zugleich im Repertoire dieser Person befinden. Dann, so die Idee der beiden Autoren, kann diese kontextuell gegebene Art und Weise zu handeln der fraglichen Person als geeignet zugeschrieben werden. So gesehen ist es dann gleichbedeutend zu sagen: „Hannah kann Fahrrad fahren.“ und: „Hannah weiß, dass diese Art und Weise, Fahrrad zu fahren, für sie eine geeignete ist. ${ }^{\text {"54 }}$ Vollständig ausformuliert lautet diese Gleichsetzung von Können und Wissen: „Relative to a context (...) [Hannah knows how to ride a bicycle] if and only if, for some contextually relevant way $w$ which is a way for Hannah to ride a bicycle, Hannah knows that $w$ is a way for her to ride a bicycle. “55

Hier nun wird Können erstaunlich genau spezifiziert. Ich halte dies allerdings für zu restriktiv. Schließlich ist Können mehr als das Äquivalent zu einer ganz bestimmten, konkreten Situation. Wenn Hannah Fahrrad fahren kann, so auch dann, wenn es niemand anderes in ihrer Umgebung auf dieselbe Art und Weise tut. Sie kann dies unabhängig von einer Situation, in der ihre spezielle Art und Weise, dies zu tun, einer weiteren, kontextuell gegebenen Art und Weise entspricht. Wenn Können mit Wissen vergleichbar ist, dann dahingehend, dass beide etwas bezeichnen, was übergreifend und unabhängig von bestimmten Kontexten gilt. Daher wäre der Rückgriff auf Art und Weisen zu handeln für die Rekonstruktion von Können nur dann gerechtfertigt, wenn es dabei nicht zu einer derart engen Zuspitzung käme. Da aber das Spektrum dessen, was Können sein kann, größer ist als eine einzelne, aufwendig rekonstruierte Art und Weise zu handeln, muss diese Methode scheitern.

\footnotetext{
${ }^{54}$ Vgl. erneut Anm. 46.

${ }^{55}$ Stanley/Williamson (2001), 426. (Meine Ergänzung, A.S.)
} 


\subsection{Können als Wissen auf eine praktische Art des Gegebenseins}

Ziel der Überlegungen Stanleys und Williamsons ist die Gegenüberstellung zweier gleichbedeutender Sätze, von denen der eine propositionales Wissen und der andere Können zum Ausdruck bringt. Die Synonymie der beiden Sätze wollen sie mit Hilfe eines Analogieschlusses belegen. Dazu stellen sie diesem Satzpaar ein weiteres an die Seite. ${ }^{56}$ Die Idee, Können lasse sich als Wissen von Art und Weisen zu handeln begreifen, hat für diese Gegenüberstellung vorbereitende Funktion. Ich akzeptiere sie einstweilen des Arguments wegen. Hier nun kommt auch wie angekündigt Freges Idee von unterschiedlichen „Arten des Gegebenseins“ ins Spiel. Sie ist zugleich der Dreh- und Angelpunkt in Stanleys und Williamsons Argumentation. Kurz gesagt ist ihre These die, dass Können Wissen auf eine praktische Art des Gegebenseins ist.

Sehen wir uns die erwähnten Sätze noch einmal an. Es klingt plausibel, dass sich die beiden Nebensätze in (1) und (2) auf dieselbe Person - nämlich John - beziehen, obwohl dafür unterschiedliche Ausdrücke verwendet werden. Es leuchtet ein, dass es sich hierbei um die gleiche Proposition handelt. Ob es sinnvoll ist, den Unterschied zwischen „dieser Mann“ und „er selbst“ auf einen Unterschied in der Art des Gegebenseins der Proposition zurückzuführen, sei dahingestellt. ${ }^{57}$ Stanley und Williamson behaupten jedenfalls, dass ein Beweis für derartige Entitäten zwar nicht einfach, aber möglich ist. ${ }^{58}$ Einen solchen oder einen Hinweis auf einen solchen bleiben sie allerdings schuldig.

Analog zu Johns Fall soll es sich nun mit (3) und (4) verhalten. Auch hier sehen die beiden Autoren lediglich einen Unterschied in der Art des Gegebenseins. Die eine bezeichnen sie als eine „demonstrative“, die andere als eine ,praktische Art des Gegebenseins“. Die Existenz letzterer erklärt sich für sie folgendermaßen: Propositionen treten grundsätzlich auf eine spezifische Art des Gegebenseins auf. Dies ist in den Beispielsätzen (1) und (2) evident. Hier liegt der Unterschied der Art des Gegebenseins in den beiden Pronomen, die darin verwendet werden. Dem Demonstrativpronomen in (1) steht das Reflexivpronomen in (2) gegenüber. Daraus

\footnotetext{
${ }^{56}$ Vgl. erneut Anm. 45 u. 46.

${ }^{57}$ Zur Problematik von „Arten des Gegebenseins“ vgl. Schiffer (1990).

${ }^{58}$ Vgl. Stanley/Williamson (2001), 429.
} 
lassen sich die „demonstrative“ und die „Erste Person Art des Gegebenseins" ableiten. In Hannahs Fall nun verhält es sich analog. Der Unterschied liegt lediglich darin, dass in (4) nicht auf die erste Person, sondern auf eine Art Praxis Bezug genommen wird. Daher handelt es sich hier um die ,praktische Art des Gegebenseins“".

Die Frage ist nun aber, ob diese Analogie überhaupt besteht. ${ }^{59}$ Denn was im einen Fall durchaus nachvollziehbar ist, ist es im anderen keineswegs. Im Beispiel mit Johns brennender Hose haben beide Sätze dieselbe propositionale Struktur. Lediglich was das Pronomen angeht weichen sie geringfügig voneinander ab. Im Beispiel mit Hannah dagegen handelt es sich um zwei völlig unterschiedliche Sätze. Anders als Stanley und Williamson behaupten, gibt es keinerlei Anhaltspunkte, die auf eine Übereinstimmung in der Satzstruktur schließen lassen. Dass es sich bei dem englischen Ausdruck „know how“ um eine versteckte Proposition in Form einer indirekten Frage handelt, hat sich zuvor als Trugschluss erwiesen. Auch die Rückführung von Können auf Wissen von Propositionen, die Art und Weisen $\mathrm{zu}$ handeln enthalten, die wiederum kontextuell gegeben sein müssen und auf die mittels Demonstrativpronomen Bezug genommen wird, vermochte nicht $\mathrm{zu}$ überzeugen. Nur wenn wir diese Vorüberlegungen hinzuziehen, lässt sich so etwas wie eine Koreferenz der beiden Sätze behaupten. Da aber weder der Rückgriff auf indirekte Fragen noch der auf Art und Weisen zu handeln Anspruch auf Gültigkeit hat, bleibt die Analogie zwischen den beiden Satzpaaren reine Spekulation.

Dies gilt aber auch in einer anderen Hinsicht. Denn der Aspekt der Indexikalität, der bei der Unterscheidung von (1) und (2) von zentraler Bedeutung ist, kommt in Stanleys und Williamsons Erklärung nicht vor. ${ }^{60}$ Schließlich können Überzeugungen, die durch einen indexikalischen Satz ausgedrückt werden, ganz andere Handlungen hervorrufen als solche, die durch einen Demonstrativsatz ausgedrückt werden. Die Überzeugung, dass „ich selbst eine brennende Hose habe“ beispielsweise, kann mich veranlassen, das Feuer umgehend zu löschen. Dagegen veranlasst mich die Überzeugung, dass „,dieser Mann eine brennende Hose hat“, dazu, Hilfe zu

${ }^{59} \mathrm{Vgl}$. auch für das Folgende Noë (2005), 288.

${ }^{60} \mathrm{Vgl}$. auch für das Folgende Jung/Newen (2010), $120 \mathrm{f}$. 
holen. Diesem Unterschied in der kausalen Rolle soll zwar die Einführung der „praktischen Art des Gegebenseins“ Rechnung tragen. Die Autoren erläutern diese Besonderheit von propositionalem und auf ,praktische Art gegebenem“ Wissen allerdings nicht weiter, sondern nehmen sie stillschweigend als gegeben an.

Schließlich ist ihre Erklärung auch noch in einem anderen Punkt unvollständig. Denn es lässt sich zeigen, dass die unspezifische Rede von „praktischen Arten des Gegebenseins“ letztlich nur eine Umschreibung von Fähigkeiten ist. ${ }^{61}$ Tatsächlich besteht nämlich der einzige erklärende Hinweis, den die beiden Autoren geben, in dem Bezug auf Dispositionen. Demnach schreiben wir jemandem, dem wir Wissen auf eine „praktische Art des Gegebenseins“ zuschreiben, bestimmte „komplexe dispositionale Zustände“ zu. ${ }^{62}$ Mit diesem Hinweis aber droht einerseits die Art von Können ins Spiel zu kommen, die ich zuvor als irrelevant ausgeschlossen habe. Können im Sinne von ,,in der Lage sein, etwas zu tun“, ist hier nicht gemeint. Schließt man diese Möglichkeit aus, so bleibt andererseits nur noch übrig, die Rede von ,auf eine praktische Art gegebenem“ Wissen als die von Fähigkeiten aufzufassen. Damit aber verfehlen die Autoren ihr eigentliches Beweisziel. Denn das bestand ja gerade darin, Können als eine Art propositionales Wissen auszuweisen. Bei Fähigkeiten kommen Propositionen allerdings nicht vor.

Stanley und Williamson müssen eine unabhängige Erklärung dafür, dass (4) eine Proposition auf eine praktische Art des Gegebenseins enthält, schuldig bleiben. Das bedeutet, dass ihre Argumentation letztlich zirkulär ist. $^{63}$ Denn die Annahme einer Analogie auf der einen Seite und einer praktischen Art des Gegebenseins auf der anderen bedingen sich gegenseitig. Unabhängig davon findet sich keine Begründung. Die Analogie zwischen (1) und (2) einerseits und (3) und (4) andererseits besteht nur so lange, wie es Propositionen auf eine praktische Art des Gegebenseins gibt. Und Propositionen gibt es genau so lange auf eine praktische Art des Gegebenseins, wie eine Analogie zwischen diesen Satzpaaren es plausibel erscheinen lässt. Andere Anhaltspunkte für diesen

\footnotetext{
${ }^{61} \mathrm{Vgl}$. Rosefeldt (2004), 375.

${ }^{62} \mathrm{Vgl}$. Stanley/Williamson (2001), $429 \mathrm{f}$.

${ }^{63} \mathrm{Vgl}$. Noë (2005), $287 \mathrm{f}$.
} 
Zusammenhang gibt es nicht. Damit aber stellt sich die gesamte Argumentation für die intellektualistische Grundannahme als eine Petitio principii heraus. Können soll eine Art des Wissens sein, weil es sich als Wissen auf eine praktische Art des Gegebenseins verstehen lässt. Können lässt sich aber nur deshalb als Wissen auf eine praktische Art des Gegebenseins verstehen, weil angenommen wird, dass es eine Art des Wissens ist. Für ihre propositionale Erklärung von Könnenssätzen setzen Stanley und Williamson also bereits voraus, dass es eine entsprechende Art des Gegebenseins gibt. Nur so können sie behaupten, dass Können Wissen ist.

Es bleibt also dabei, dass der Intellektualismus auch in der Lesart Stanleys und Williamsons keine kohärente Erklärung für das Verhältnis von Wissen und Können darstellt. Ihr aufwendiger Rehabilitierungsversuch führt letzten Endes nicht $\mathrm{zu}$ dem erwünschten Ergebnis. Der Intellektualismus bleibt widerlegt. Ryles Kritik hat also weiterhin Bestand. Sein Regressargument bleibt weiterhin gültig. Damit verlagert sich auch die Frage, ob Wissen und Können in dieser Erklärung als vollkommen kongruent betrachtet werden kann, ins Hypothetische. So wenig sich diese Version des Intellektualismus belegen lässt, so wenig lässt sich ein Kongruenzverhältnis etablieren. Zuvor schon hat sich gezeigt, dass sich das Verhältnis von Wissen und Können sinnvollerweise weder als kongruent noch als exklusiv beschreiben lässt. Denn für einen koextensionalen Bezug zu einem gemeinsamen Gegenstand lässt sich kein geeigneter Kandidat finden. Und gegen eine strikte Trennung der beiden Begriffe spricht die Tatsache, dass eine Umschreibung von Können in Form von propositionalem Wissen zumindest teilweise möglich sein kann. Somit bleibt die anti-intellektualistische Auffassung, dass Wissen eine Art des Könnens ist, als die plausibelste Beschreibung dieses Verhältnisses übrig. Sie ist das Thema des verbleibenden Teils dieser Arbeit. 